\title{
Hypnosis and cortisol: the odd couple
}

\begin{abstract}
This review is focused on the hypothesis that hypnosis can differentially modulate T-cell subsets and that this effect is mediated by changes in hypothalamo-pituitary-adrenal (HPA) mediators, such as cortisol. Psychoneuroimmunology (PNI) is the study of the interaction between psychological processes and the nervous and immune systems, taking an interdisciplinary approach including psychology, neuroscience, immunology, physiology, genetics, pharmacology, molecular biology, psychiatry, behavioral medicine, infectious diseases, endocrinology and rheumatology. The main interests of this discipline are the interactions between the nervous and immune systems studying mental processes leading to pathologies due to auto-immunity or immune deficiency. Here we make an overview about history of PNI focusing on hypnosis as a modulator of immune response and a therapeutic tool to heal stress related diseases. Here we also share our ideas about studying clinical use of cortisol as a reliable monitor for hypnosis therapy on the homeostasis of hypothalamicpituitary-adrenal axis (HPA axis)
\end{abstract}

Keywords: psychoneuroimmunology, cortisol, stress, lymphocytes, hypnosis
Volume I Issue 2 - 2014

Mario Scardino Scardino,' Antonio Scardino

'Department of General Medicine, Civil Hospital of Urbino, Italy ${ }^{2}$ Clinical Psychology, University Hospital Umberto I, Italy

Correspondence: Antonio Scardino, Department of General Medicine, Civil Hospital of Urbino, Via Comandino, 70- 61029Urbino, Italy, Tel 39-0722-30I I II,

Email dr.antonio.scardino@gmail.com

Received: May 19, 2014 | Published: June 12, 2014
Abbreviations: HPA, hypothalamo-pituitary-adrenal; PNI, psychoneuroimmunology; HPA axis, hypothalamic-pituitary-adrenal Axis; DCs, dendritic cells

\section{Introduction}

Interest in the relationship between psychiatric syndromes and immune function has been a consistent theme since the beginning of modern medicine. Claude Bernard, a French physiologist of the Museum national d'Histoire naturelle, founded the concept milieu interieur in the mid-1800s. Walter Cannon, a professor of physiology at Harvard University coined the commonly used term homeostasis in his book The Wisdom of the Body, 1932, from the Greek word homoios, meaning similar, and stasis, meaning position. In his work with animals. Cannon observed that any change of emotional state in the beast, such as anxiety, distress, or rage was accompanied by total cessation of movements of the stomach (Bodily Changes in Pain, Hunger, Fear and Rage, 1915). Hans Selye, a student of Johns Hopkins experimented physical and mental adverse conditions and noted that the body consistently adapted to heal and recover forming the empiric foundation of the General Adaptation Syndrome, consisting of an enlargement of the adrenal gland, atrophy of the thymus, spleen and other lymphoid tissue, and gastric ulcerations. This foundational work led to a rich line of research on the biological functioning of glucocorticoids. ${ }^{1,2}$ Mid 20th century studies of psychiatric patients reported immune alterations in psychotic patients, including numbers of lymphocytes ${ }^{3,4}$ and poorer antibody response to pertussis vaccination, compared with non psychiatric control subjects..$^{5}$ In 1964 George F. Solomon, of The University of California in Los Angeles et al. coined the term psychoimmunology and published a landmark paper: Emotions, immunity, and disease: a speculative theoretical integration. ${ }^{6}$ In 1975 Robert Ader and Nicholas Cohen, a psychologist and an immunologist at the University of Rochester, advanced PNI with their demonstration of classic conditioning of immune function in animals and coined the term psychoneuroimmunology. ${ }^{7,8}$ Ader, Cohen and Felten went on to edit the groundbreaking book Psychoneuroimmunology in 1981, which laid out the underlying premise that the brain and immune system represent a single, integrated system of defense. In 1985, research by neuropharmacologist Candace Pert, of the National Institutes of
Health at Georgetown University revealed that neuropeptide-specific receptors are present on the cell walls of both the brain and the immune system. ${ }^{9,10}$ Contemporary advances in psychiatry, immunology, neurology and other integrated disciplines of medicine has fostered enormous growth for PNI. At present time, evidence for nervous system-immune system interactions exists at several biological levels and we know that nerves in the thymus and spleen terminating near clusters of lymphocytes, macrophages and mast cells, all of which help control immune function..$^{11-14}$ The brain and the immune system are the two major adaptive systems of the body and they talk to each other through signaling pathways. The major pathway involved in this cross-talk is throughout hypothalamic-pituitary-adrenal axis. The HPA axis responds to physical, environmental and mental challenge to maintain homeostasis in part by controlling the body's cortisol level. ${ }^{15-18}$

\section{T lymphocytes and hypnosis}

T-lymphocytes are a central component of the adaptive immune response, since these cells have many functions, including antigen recognition, regulation of antibody and cell-mediated immunity, as well as effector functions, e.g. killing of virally-infected or neoplastic target cells. ${ }^{19-21}$ Moreover, T-cells have been implicated in the hypnosis and differential cytokine expression pathogenesis of an auto-immune and infectious diseases including rheumatoid arthritis, diabetes mellitus, AIDS, tuberculosis, and periodontitis. Consequently, the ability to modulate the T-cell response will be of interest in the maintenance of health as well as the treatment of disease states. Hypnosis has evidenced significantly elevated total lymphocyte counts and also significantly increased total number of T-cells in high hypnotizable subjects. ${ }^{22,23}$ Furthermore, a combination of hypnosis, progressive muscle relaxation, biofeedback relaxation, and meditation has been demonstrated to induce an increase in T-cells in HIV-positive subjects with low T-cell counts. ${ }^{24}$ Traditionally, T-cells have been classified into two subsets of cells, based on the expression of CD4 and CD8 cell surface molecules, where T-cells expressing interleukin (IL-2) and interferon gamma (IFN-Y) belong to the inflammatory subset that regulate cell-mediated immunity and are known as Type 1 helper (Th1) cells. Actually, CD4+ T-helper (Th) cells are a major cell population that plays an important role in governing acquired immune responses to a variety of foreign antigens as well as inducing 
some types of autoimmune diseases. There are at least four distinct Th cell subsets (Th1, Th2, Th17, and inducible T-regulatory cells), each of which has specialized functions to control immune responses. Each of these cell types emerge from naive CD4+ $\mathrm{T}$ cells after encounter with foreign antigens presented by dendritic cells (DCs) ${ }^{25}$. There is evidence to suggest that the various subsets of T-cells are differentially modulated by hypothalamo-pituitary-adrenal (HPA) axis mediators. ${ }^{26-28}$ Other evidences show that circadian variations in $\mathrm{T}$ cell subpopulations in human blood are differentially regulated via release of cortisol and catecholamines: within the CD4(+) and CD8(+) $\mathrm{T}$ cell subsets, naive cells show pronounced circadian rhythms with a daytime nadir, whereas effector $\mathrm{CD} 8(+) \mathrm{T}$ cell counts peak during daytime. Furthermore, naive $\mathrm{T}$ cells were negatively correlated with cortisol rhythms, decreased after low-dose cortisol infusion, and showed highest expression of CXCR4, which was up-regulated by cortisol. ${ }^{29,30}$. A significant decrease in the percentage of CD4(+) T cells and increase in the percentage of CD8(+) T cells were found in patients with Systemic Lupus Erythematosus compared to the healthy controls. ${ }^{31}$ Hence, it is reasonable to hypothesize that modulation of neuro-endocrine mediators (such as cortisol) by hypnosis is likely to have different effects on Th1 and Th2 cells. This potential shift of T-cell activity can have a potentially significant influence on diseases in which T-cells have been implicated. ${ }^{32-35}$ Documentation of a neuroendocrine response to hypnosis is limited and yet controversial. For example, a recent study from Varga K. and Kekecs Z. shows that the changes in oxytocin and cortisol levels after hypnosis are not related to hypnotic susceptibility but to relational experiences. ${ }^{36}$ Nevertheless, cortisol has been shown to decrease to very low levels during extended hypnosis onto highly hypnotizable subjects. ${ }^{37,38}$ Significant differences in the levels of cortisol were also found in highly hypnotizable subjects when they re-experienced intense emotional states induced under hypnosis. ${ }^{22-23}$ Cortisol was significantly lower following the last suggested emotional state of happiness in comparison with the emotional state before hypnosis or the preceding emotional states of anger and depression. Although very limited, this evidences suggest that hypnosis has the potential to modulate the HPA axis. On the other hand, the clinical significance of hypnosis to alter the T-cell response is far to be completely clear. It is under current study how hypnosis leads to a modulation of cortisol and a subsequent decrease in the Th1 subset of T- cells; this intervention may potentially be used in clinical therapy involving auto-immune diseases or in the pathologies where the over expression of Th1 cells occurs, such as rheumatoid arthritis or insulin dependent diabetes mellitus. Statistically significant effects of hypnosis on cortisol levels and immune response are our current challenges.

\section{Hypnotherapy}

There has been an increasing interest about the link between interpersonal, social, behavioral stressors and health. Many groups are trying to clarify the best techniques to interact with the immunity throughout the cognitive or the unconsciousness evoked by relaxation or hypnosis, considering blood's white cells and their receptors as a sort of circulating portion of the nervous system. One of the techniques to interact with effects of stressors on human body is hypnotherapy. ${ }^{39,40}$ Hypnosis is considered a psychosomatic event normally preceded by a hypnotic induction technique. There are several different induction techniques, among them one of the most used methods has been refined by the psychiatrist Milton H. Erickson, in terms of unconventional approach to psychotherapy, as described in his works and books. He developed an extensive use of therapeutic metaphor and story as well as hypnosis and coined the term brief therapy for his method of addressing therapeutic change in relatively few sessions. Through conceptualizing the unconscious as highly separate from the conscious mind, with its own awareness, interests, responses, and learnings, he taught that the unconscious mind was creative, solutiongenerating, and often positive. ${ }^{41,42}$ But the main question remains unsolved: how can we actually measure human stress? The question is far to be trivial. Measuring the stress level by a quantifiable, scientific tool might discriminate effective or ineffective therapeutic techniques currently used to heal stressors related to human diseases.

\section{Hypnosis and cortisol: the odd couple}

The use of cortisol during hypnotherapy is our model for studying non-pharmacological modulation of the immune response and the cellular and molecular mediators involved. The cortisol levels in saliva can be checked out by new devices (salivettes) in every place, at home, in a stress free condition as well as prisons, boats, airplanes, opening the possibility to monitoring real time unconventional techniques, such as hypnosis, for their efficacy. Finally, blood samples might display lymphocytes rate and activation, plasma cortisol, ACTH or endorphins at different time points of a long term therapy. Thus, the question is: can hypnosis cure people? We are currently using the hypnosis-cortisol model for the comprehension of effectiveness of hypnotherapy technique. At present time, we are studying cortisol as a reliable indicator of therapeutic adequacy of hypnotherapy to the modulation of immune response in patients suffering of auto-immune diseases. We are confident that the experiments currently in progress will clarify our doubts.

\section{Acknowledgements}

None.

\section{Conflicts of interest}

The authors declare that there are no conflicts of interest

\section{Funding}

None.

\section{References}

1. Michael RI, Kavita V. Human Psychoneuroimmunology. 1st edn. USA: Oxford University Press; 2005. pp. 368.

2. Thomas CN. Hans Selye and the Field of Stress Research. The Journal of Neuropsychiatry and Clinical Neurosciences. 1998;10:230-230.

3. Freeman H, Elmadjian F. The relationship between blood sugar and lymphocyte levels in normal and psychotic subjects. Psychosomatic Medicine. 1947;9(4):226-33.

4. Phillips L, Elmadjian F. A Rorschach tension score and the diurnal lymphocyte curve in psychotic subjects. Psychosom Med. 1947;9(6):364-371.

5. Vaughan WTJ, Sullivan JC, Elmadjian F. Immunity and schizophrenia a survey of the ability of schizophrenic patients to develop an active immunity following the injection of pertussis vaccine. Psychosom Med. 1994;11(6):327-333.

6. Solomon GF, Moos RH. Emotions, immunity, and disease:a speculative theoretical integration. Arch Gen Psychiatry. 1964;11:657-674.

7. Ader R, Cohen N. Behaviorally conditioned immunosuppression. Psychosom Med. 1975;37(4):333-340.

8. https://www.urmc.rochester.edu/libraries/miner/historical_services/ archives/Faculty/PapersofRobertAder.cfm 
9. Pert CB, Ruff MR, Weber RJ, et al. Neuropeptides and their receptors:a psychosomatic network. J Immunol. 1985;135(2 Suppl):820s-826s.

10. Miller GE, Chen E, Zhou ES. If it goes up, must it come down? Chronic stress and the hypothalamic-pituitary-adrenocortical axis in humans. Psychol Bull. 2007;133(1):25-45.

11. Covelli V, Passeri ME, Leogrande D, et al. Drug targets in stress-related disorders. Curr Med Chem. 2005;12(15):1801-1809.

12. Elenkov IJ, Iezzoni DG, Daly A, et al. Cytokine dysregulation, inflammation and well-being. Neuroimmunomodulation. 2005;12(5):255-269.

13. Kaprio J, Koskenvuo M , Rita H. Mortality after bereavement:a prospective study of 95,647 widowed persons. Am J Public Health. 1987;77(3):283-287.

14. Chrousos GP and Gold PW. The concepts of stress and stress system disorders. Overview of physical and behavioral homeostasis. JAMA. 1992;267(9):1244-1252.

15. Kiecolt-Glaser JK. Handbook of Human Stress and Immunity. USA San Diego Academic Press; 1994.

16. Cohen S, Tyrrell DA, Smith AP. Psychological stress and susceptibility to the common cold. The N Engl J Med. 1991;325(9):606-612.

17. Cohen S and Williamson GM. Stress and infectious disease in humans Psychol Bull. 1991;109(1):5-24.

18. Leserman J, Petitto JM, Golden RN, et al. Impact of stressful life events, depression, social support, coping, and cortisol on progression to AIDS. Am J Psychiatry. 2000;157(8):1221-1228.

19. Scardino A, Alimandi M, Correale P, et al. A polyepitope DNA vaccine targeted to Her-2/ErbB-2 elicits a broad range of human and murine CTL effectors to protect against tumor challenge. Cancer Res. 2007;67(14):7028-7036.

20. Bei R, Scardino A. TAA polyepitope DNA-based vaccines:a potential tool for cancer therapy. J Biomed Biotechnol. 2010:102758.

21. Scardino A, Paroli M, De Petrillo G, et al. Antigen targeting to antigen-presenting cells enhances presentation to class II-restricted T lymphocytes. Immunology. 1994;81(1):167-170.

22. Hall HR. Hypnosis and the immune system:a review with implications for cancer and the psychology of healing. Am J Clin Hypn. 1983;25(23):92-103.

23. Ruzyla SP, Barabasz A, Barabasz M, et al. Effects of hypnosis on the immune response:B-cells, T-cells, helper and suppressor cells. Am J Clin Hypn. 1995;38(2):71-79.

24. Taylor DN. Effects of a behavioral stress-management program on anxiety, mood, self-esteem, and T-cell count in HIV positive men. Psychol Rep. 1995;76(2):451-457.

25. Yamane H, Paul WE. Early signaling events that underlie fate decisions of naive CD4(+) T cells toward distinct T-helper cell subsets. Immunol Rev. 2013;252(1):12-23.
26. Wood GJ, Bughi S, Morrison J, et al. Hypnosis, differential expression of cytokines by T-cell subsets, and the hypothalamo-pituitary-adrenal axis. Am J Clin Hypn. 2003;45(3):179-196.

27. Zachariae R, Bjerring $\mathrm{P}$, Zachariae $\mathrm{C}$, et al. Monocyte chemotactic activity in sera after hypnotically induced emotional states. Scand J Immunol. 1991;34(1):71-79.

28. Leserman, J, Jackson ED, Petitto JM,et al. Progression to AIDS:the effects of stress, depressive symptoms, and social support. Psychosom Med. 1999;61(3):397-406.

29. Dimitrov S, Benedict C, Heutling D, et al. Cortisol and epinephrine control opposing circadian rhythms in $\mathrm{T}$ cell subsets. Blood. 2009;113(21):5134-5143.

30. Bollinger T1, Bollinger A, Naujoks $J$, et al. The influence of regulatory T cells and diurnal hormone rhythms on Thelper cell activity. Immunology. 2010;131(4):488-500.

31. Shah D1, Kiran R, Wanchu A, et al. Relationship between T lymphocyte subsets and cortisol in systemic lupus erythematosus. Kathmandu Univ Med J (KUMJ). 2009;7(27):213-219.

32. Andersen BL, Kiecolt-Glaser JK, Glaser R. A biobehavioral model of cancer stress and disease course. Am Psychol. 1994;49(5):389-404.

33. Kiecolt-Glaser JK and Glaser R. Psychoneuroimmunology and cancer: fact or fiction? Eur J Cancer. 1999;35(11):1603-1607.

34. McDonald RD, Yagi K. A note on eosinopenia as an index of psychological stress. Psychosom Med. 1960;22(2):149-150.

35. Herbert TB, Cohen S (1993) Stress and immunity in humans:a metaanalytic review. Psychosom Med. 1993;55(4):364-379.

36. Varga K, Kekecs Z. Oxytocin and cortisol in the hypnotic interaction. Int J Clin Exp Hypn. 2014;62(1):111-128.

37. Sachar EJ, Fishman JR, Mason JW. Influence of the hypnotic trance on plasma 17-hydroxycorticosteroid concentration. Psychosom Med. $1965 ; 27: 330-341$

38. Sachar EJ, Cobb JC, Shor RE. Plasma cortisol changes during hypnotic trance. Relation to depth of hypnosis. Arch Gen Psychiatry. 1996;14(5):482-490.

39. Amelio RD, Scardino M, Gsell E, et al. The Mother as the Child's First Family:I - Hypnosis Cognitively Integrated with Group Analysis. The international Journal of Prenatal and Perinatal Studies. 1992;4:87-195.

40. Amelio RD, Scardino M, Gsell E, et al. The Mother as the Child's First Family:II - Regression under Hypnosis. The international Journal of Prenatal and Perinatal Studies. 1992;4:197-203.

41. Erickson MH. Further clinical techniques of hypnosis:utilization techniques 1959. Am J Clin Hypn. 2009;51(4):341-362.

42. Erickson MH. Naturalistic techniques of hypnosis 1958. Am J Clin Hypn. 2009;51(4):333-340. 